\title{
Ajustamento Mental ao Cancro do Pulmão: o papel da autocompaixão e do suporte social
}

\author{
Psychological Adjustment to Lung Cancer: the role of self-compassion and social support
}

Artigo Original | Original Article

Rute Batista, Psy M (1a) Marina Cunha, PhD (1,2b) Ana Galhardo, PhD (1,2c) Margarida Couto, PhD (1 c)

(1) Instituto Superior Miguel Torga, Coimbra, Portugal

(2) Centro de Investigação do Núcleo de Estudos e Intervenções Cognitivo-comportamentais; Faculdade de Psicologia e de Ciências da Educação, Universidade de Coimbra, Coimbra, Portugal.

(a) Recolheu e inseriu os dados para análise estatística e deu o maior contributo para a elaboração do trabalho.

(b) Deu o maior contributo, logo a seguir ao primeiro autor, para a elaboração do trabalho; conduziu a maioria das análises estatísticas; reviu o trabalho.

(c) Contribuiu significativamente para a revisão do trabalho

Autor para correspondência | Corresponding author: Rute Damas Batista, rutedamasbatista@hotmail.com

Palavras-Chave

Cancro do pulmão

Ajustamento mental

Psicopatologia

Autocompaixão

Suporte social

\section{RESUMO}

Objetivo: É bem conhecido o impacto que o diagnóstico de uma doença oncológica tem ao nível do ajustamento psicológico em doentes com cancro do pulmão. Por outro lado sabe-se que a sintomatologia depressiva pode, também, sobrepor-se aos sintomas físicos do cancro e tratamento oncológico, o que dificulta a sua deteção e adequada abordagem terapêutica.

O presente trabalho pretende explorar em que medida a autocompaixão e o suporte social são preditores do ajustamento mental e estados afetivos negativos em doentes com cancro do pulmão.

Método: A amostra é constituída por 55 indivíduos (38 homens e 17 mulheres) diagnosticados com cancro do pulmão e com idades compreendidas entre os 44 e os 87 anos. Como instrumentos de medida foram utilizadas a Escala de Ajustamento Mental ao Cancro (MiniMac), a Escala de Autocompaixão (SELFCS), a Escala de Satisfação com o Suporte Social (ESSS) e a Escala de Ansiedade, Depressão e Stress (EADS-21).

Resultados: Foram encontradas correlações significativas entre o ajustamento mental, a psicopatologia e as estratégias de regulação emocional (autocompaixão) e suporte social. Os modelos preditores do ajustamento mental e da sintomatologia associada ao stress incluem dimensões da autocompaixão e o suporte social como variáveis preditoras significativas. Já em relação ao modelo preditor da sintomatologia depressiva, o mindfulness parece ser a única variável com um contributo relevante.

Conclusões: Estes resultados têm implicações práticas, sugerindo que estes doentes podem no seu programa terapêutico beneficiar do desenvolvimento deste tipo de estratégias (novas formas de se relacionarem com as suas experiências emocionais e qualidade das suas redes sociais) no sentido de promover um melhor ajustamento mental à sua condição.

\section{ABSTRACT}

Introduction: The impact of the diagnosis of an oncologic disease is well-known in terms of psychological adjustment and quality of life. On the other hand it is known that depressive symptoms may also overlap the physical symptoms of cancer and cancer treatment, which may interfere in their detection and appropriate treatment approach.

Objectives: The aim of the current study was to explore the relationship between psychological adjustment to lung cancer, self-compassion, social support and emotional negative states in patients with lung cancer.

Method: Fifty-five patients diagnosed with lung cancer ( 38 men and 17 women) with ages ranging from 44 to 87 years old participated in the study. A set of self-report instruments was used: the Mini Mental Adjustment to Cancer Scale (MiniMac), the Self-compassion Scale (SCS; Neff, 2003), the Social Support Satisfaction Scale (SSSS) and the Depression, Anxiety and Stress Scale (DASS-21).

Results: Significant correlations were found between psychological adjustment, psychopathology, emotion regulation strategies (self-compassion), and social support. The predictive models for psychological adjustment and stress related symptomatology include self-compassion and social support as significant predictive variables. Regarding the predictive model for depressive symptomatology, mindfulness seems to be the only significant predictor.

Conclusions: Our findings suggest that these patients may benefit, in their therapeutic approach, from the development of this kind of strategies (new ways of relating themselves with their emotional experiences and quality of their social networks) in order to promote a better psychological adjustment to their clinical condition. 


\section{INTRODUÇÃO}

O cancro é uma doença de origem genética causada por mutações do ácido desoxirribonucleico (ADN). Existem mais de 200 tipos de cancro, entre os quais os mais incidentes são os da mama, do pulmão, do colorretal e da próstata (Bower e Waxman 2006).

De doença rara no passado, o cancro do pulmão transformou-se na doença neoplásica mais comum e mais mortal, em todo o mundo (Zamboni, 2002), sendo, a nível mundial, responsável por 1,8 milhões de novos casos em 2012 (Ferlay et al., 2008). Em Portugal, o cancro do pulmão é considerado a principal causa de morte por cancro no sexo masculino (Ferlay et al., 2008). Menos frequente no sexo feminino, corresponde apenas a uma taxa de $20 \%$ do número total de casos, verificando-se contudo, nos últimos anos, uma tendência para aumentar (Alves, Bastos e Lunet, 2009). De realçar também que a taxa de incidência de cancro do pulmão tem vindo a crescer, independentemente da etnia, condições socioeconómicas ou geográficas (Ferlay et al., 2008; Parente et al., 2007).

Apesar das causas do cancro do pulmão serem predominantemente ambientais, é provável que exista uma variação individual substancial na suscetibilidade aos carcinogénios respiratórios. O risco de neoplasia pode ser conceptualizado como reflexo das consequências conjuntas e da inter-relação entre a exposição aos agentes etiológicos ou protetores e a suscetibilidade individual a esses agentes (Alberg e Samet, 2003). Tendo em conta a etiologia multifatorial do cancro do pulmão, as interações sinérgicas entre os vários fatores de risco podem ter consequências substanciais para o desenvolvimento da doença, referindo-se como exemplo o sinergismo entre hábitos tabágicos e a exposição ao amianto.

Relativamente ao tratamento desta doença, em quase todos os tipos de cancro existem opções de tratamento consideradas eficazes, entre elas a cirurgia, a quimioterapia e a radioterapia, devendo o tratamento adaptar-se a cada caso, de acordo com a realidade de cada paciente (Ogden, 1999; Straub, 2005). Na revisão da literatura é amplamente reconhecido que os doentes oncológicos, ao longo de todo o percurso associado à doença (diagnóstico, tratamento e follow-up), desenvolvem problemas a nível psicológico, sendo por vezes frequente o aparecimento de sintomas de ansiedade e de depressão com relevância clínica, assim como uma diminuição na sua qualidade de vida (Santos e Ribeiro, 2001; Marques et al., 1991).

\section{Sofrimento psicológico e cancro do pulmão}

O doente com patologia oncológica sofre mudanças, designadamente físicas (alterações da imagem corporal), psicológicas, sociais (perda de papéis) e espirituais (perda do sentido da vida) (Pereira e Lopes, 2005). Entre as alterações psicológicas mais evidentes, Pereira e Lopes (2005) apontam a ansiedade, a depressão, a expressão de sentimentos de medo, a raiva, a revolta, a desesperança, a culpa e os sentimentos suicidas. Stommel, Kurtz, Kurtz, Given e Given (2004) encontraram nos seus estudos valores de depressão mais elevados em doentes com cancro do pulmão comparativamente a doentes com cancro da mama, do cólon e da próstata. Este facto constitui um pesado fator de risco, dado que a sintomatologia depressiva pode, também, sobrepor-se e/ou confundir-se com sintomas físicos do cancro e do seu tratamento, o que dificulta a deteção da depressão e adequada abordagem terapêutica (Domingues e Albuquerque, 2008).

Outro dado importante é o facto da experiência do estigma no cancro do pulmão ser moldada pela associação entre a doença e o consumo de tabaco, pela perceção da doença como uma lesão autoinfligida, pela sua elevada taxa de mortalidade e pelo tipo de morte, com grande sofrimento (Domingues e Albuquerque, 2008). Assim, segundo Domingues e Albuquerque (2008), características da doença como mau prognóstico, a culpabilidade do doente relativamente a comportamentos de risco ao longo da vida, nomeadamente tabagismo, e o estigma associado ao consumo de tabaco e cancro do pulmão são apontados como fatores responsáveis pela elevada prevalência de maior sofrimento psicológico em doentes com este tipo de cancro.

\section{Autocompaixão e saúde/doença}

Partindo da tradição Budista e da Psicologia Social, Neff (2003b) descreve a autocompaixão como uma atitude calorosa e de aceitação em relação aos aspetos negativos da self ou da vida. Segundo esta linha de pensamento, distinguem-se três componentes principais: (a) componente de calor/compreensão, isto é, a capacidade para ser amável e compreensível para consigo próprio, em vez de uma atitude demasiada crítica e punitiva; (b) componente de condição humana, que significa entender as próprias experiências como parte de uma experiência humana mais abrangente por oposição ao isolamento; e (c) componente de mindfulness, referente à consciência equilibrada e aceitação dos sentimentos e sentimentos dolorosos, sem uma excessiva sobreidentificação com os mesmos.

Este conjunto de competências associadas à (auto)compaixão tem vindo a ser estudado como um processo de regulação emocional já que pode ser traduzido por uma forma particular de lidar com 
experiências dolorosas, evidenciando um efeito protetor relativamente ao desenvolvimento de psicopatologia. $\mathrm{Na}$ verdade, os estudos de Leary, Tate, Adams, Allen e Hancock (2007) mostram como a autocompaixão é capaz de moderar as reações a acontecimentos negativos (fracasso, rejeição, vergonha), amortecendo o impacto destes acontecimentos negativos para o indivíduo. Este efeito amortecedor poderá estar também relacionado com o facto dos indivíduos mais compassivos serem menos autocríticos, fazerem avaliações mais realistas dos seus desempenhos, terem mais tolerância e compreensão pelo Eu e a autoavaliação não depender tanto da qualidade ou quantidade dos resultados obtidos.

No domínio da oncologia, o estudo de PintoGouveia, Duarte, Matos e Fráguas (2013) realizado com pacientes diagnosticados com cancro, realçou o papel relevante da autocompaixão no funcionamento psicológico destes indivíduos. Os autores verificaram que os pacientes com níveis mais elevados de autocompaixão apresentavam um melhor funcionamento psicológico, nomeadamente, uma menor sintomatologia ansiosa, depressiva e associada ao stress.

Por sua vez, a autocompaixão tem-se mostrado fortemente associada ao bem-estar psicológico, à felicidade, à satisfação com a vida, ao otimismo, à inteligência emocional e relações interpessoais (Neff, Kirkpatrick e Rude, 2007; Neff, Rude, e Kirkpatrick, 2007; Neff e Costigan, 2014). O facto das competências de autocompaixão se mostrarem associadas à capacidade de gerir a adversidade e de fazer mudanças necessárias na vida (Neff, Kirkpatrick, e Rude, 2007), pode ser particularmente relevante nos indivíduos com doença oncológica tendo em conta os desafios próprios desta condição.

\section{Suporte Social e saúde/doença}

O suporte ou o apoio social é um termo abrangente que diz respeito não só à coesão e à quantidade de relações sociais que o indivíduo estabelece, mas também à força e frequência dos laços constituídos e ao modo como tal sistema de apoio é percebido. Assim, de acordo com Stewart, Craig, MacPherson e Alexander (2001), o suporte social pode ser conceptualizado como a interação com familiares, amigos, profissionais e pares que providenciam informação, confiança, ajuda e estima.

A perceção da disponibilidade do suporte social tem estado consistentemente associada a uma melhor adaptação fisiológica e psicológica (Custódio, 2010). Na vida do adulto com cancro, as redes de apoio são benéficas quando prestam apoio de forma a influenciar positivamente a saúde (Rodrigues e Ferreira, 2012). Segundo Usta (2012), o apoio social tem uma importância extraordinária no confronto com problemas psicológicos, como ansiedade e depressão, ambas comuns em doentes com cancro.

Apesar da diversidade de definições, parece haver consenso no que se refere à importância do suporte social na diminuição dos efeitos nefastos do stress, bem como na promoção do bem-estar e qualidade de vida do indivíduo (Pais Ribeiro, 1999).

Esta multiplicidade de conceptualizações do suporte social tem-se refletido na forma como este constructo tem sido medido, originando vários instrumentos de avaliação que realçam, cada um deles, diferentes componentes ou dimensões do suporte social, sem, no entanto, o contemplar na sua totalidade. A este propósito, Pais Ribeiro (1999) desenvolveu a Escala de Satisfação com o Suporte Social a qual pretende medir o grau de satisfação com o suporte social existente, partindo do pressuposto de que "as medidas de perceção de suporte social explicam melhor a saúde do que as de suporte social tangível" (Pais Ribeiro, 1999, p. 552).

Neste enquadramento teórico, a satisfação com o suporte social refere-se a um julgamento pessoal do individuo sobre as suas necessidades de suporte, o que recebe para as satisfazer, bem como o custo estimado desse apoio. De acordo com estes autores, trata-se de uma dimensão subjetiva que é fundamental para o bemestar e qualidade de vida, tanto em populações saudáveis como doentes. No estudo da adaptação da referida escala a pessoas com doença oncológica, os dados corroboraram a aplicabilidade deste constructo teórico nas suas diferentes dimensões às características da doença oncológica (Santos, Pais Ribeiro e Lopes, 2003).

Adicionalmente, a satisfação com o suporte social mostrou-se correlacionada com o processo de adaptação à doença oncológica e aos resultados de saúde, nomeadamente a qualidade de vida. Ainda, neste estudo, não foram encontradas diferenças significativas na satisfação com o suporte social em função dos grupos de doença oncológica, o que está em consonância com a literatura que aponta o papel pouco relevante do tipo de cancro no processo de coping e seus recursos (Santos et al., 2003).

O presente estudo tem como principal objetivo avaliar o contributo (papel preditor) da autocompaixão e da satisfação com o suporte social para o ajustamento mental e consequentes sintomas emocionais negativos de doentes com cancro do pulmão. Paralelamente foi explorado se variáveis clínicas como ser ou não fumador, ter ou não antecedentes familiares oncológicos, o tempo de conhecimento do diagnóstico e a perceção da gravidade da doença poderiam afetar as estratégias de coping, o suporte social, a autocompaixão, e sintomas emocionais negativos. 


\section{MÉTODO}

\section{Participantes}

Para o presente estudo foi usada uma amostra por conveniência, recolhida no Hospital de Dia de Oncologia do Centro Hospitalar e Universitário de Coimbra.

A amostra é constituída por 55 doentes diagnosticados com cancro do pulmão, independentemente do seu estádio e da existência de comorbilidades com outras patologias orgânicas. Foram admitidos doentes de ambos os sexos, mediante o respeito pelos seguintes critérios de inclusão: a) idade superior a 18 anos; b) diagnóstico de cancro do pulmão; c) garantia de que os participantes não receberam ou estejam a receber apoio psicológico, relacionado com a doença, até à data (de forma a evitar uma contaminação dos resultados no que diz respeito ao ajustamento mental dos indivíduos à doença oncológica). Fizeram parte da amostra 38 homens (69\%) e 17 mulheres (31\%), com idades compreendidas entre os 44 e os 87 anos, sendo a média de idades de 66,36 ( $D P=9,60)$.

Relativamente ao estado civil, 5 indivíduos (9,1\%) são solteiros, $38(69,1 \%)$ são casados, $3(5,5 \%)$ são divorciados e $9(16,4 \%)$ são viúvos. No que diz respeito ao nível socioeconómico, 9 participantes $(16,4 \%)$ percecionam o seu nível como baixo, 28 (50,9\%) percecionam como médio baixo, $17(30,9 \%)$ consideram ter um nível médio e 1 indivíduo $(1,8 \%)$ considera ter um nível socioeconómico médio alto.

Quanto ao meio de residência, o mais prevalente é o meio rural $(63,2 \%$ no sexo masculino e $88,2 \%$ no sexo feminino), sendo que no meio urbano residem apenas 14 homens $(36,8 \%)$ e 2 mulheres ( $11,8 \%)$.

\section{Instrumentos}

A Escala Reduzida de Ajustamento Mental ao Cancro (The Mini-Mental Adjustment to Cancer Scale MiniMac; Watson, Greer, Inayat, Burgess e Robertson, 1994, versão portuguesa de Pais-Ribeiro, Ramos e Samico, 2003) inclui 29 itens que avaliam as estratégias de coping utilizadas pelos doentes oncológicos, isto é, a forma como os sujeitos lidam com o diagnóstico e tratamento de cancro. Os itens distribuem-se por cinco subescalas que cobrem as dimensões: DesânimoFraqueza (e.g., "Sinto que a vida não tem esperança") caracterizada pelo pessimismo e a ausência de estratégias de combate ativo da doença; Preocupação Ansiosa (e.g., "Estou preocupado que a doença volte a aparecer") caracterizada por uma ansiedade persistente; Espírito de luta (e.g., "estou determinado a vencer a minha doença”) refere-se a um padrão de resposta dominado por uma atitude otimista; Evitamento Cognitivo (e.g., "faço um esforço positivo para não pensar na minha doença") o qual remete para a recusa do conhecimento do diagnóstico; e Fatalismo (e.g., Entreguei-me nas mão de Deus") o qual diz respeito à aceitação passiva da doença. Os itens são cotados numa escala de 0 a 3 pontos, permitindo a MiniMac obter cinco pontuações referentes a cada uma das dimensões mencionadas.

$\mathrm{Na}$ versão portuguesa, validada por Pais-Ribeiro, Ramos e Samico (2003), os resultados apontaram para valores de consistência interna aceitáveis para quatro das cinco dimensões (Desânimo/fraqueza: $\alpha=0,79$; Preocupação ansiosa: $\alpha=0,88$; Evitamento cognitivo: $\alpha=$ 0,84; Espírito de Luta: $a=0,72)$, sendo a dimensão “Fatalismo" a exceção $(\alpha=0,28)$.

A Escala da Autocompaixão (Self-Compassion Scale SELFCS; Neff 2003a; versão portuguesa de Castilho e Pinto-Gouveia, 2011) é composta por 26 itens que procuram avaliar a autocompaixão do indivíduo, enquanto traço. Cada item é respondido com base numa escala de 0 a 5 pontos. Para além da pontuação total, a escala permite obter pontuações referentes aos seis fatores que a constituem: Calor/Compreensão (e.g., "Tento ser compreensivo e paciente em relação aos aspetos da minha personalidade e que não gosto"); Autocrítica (e.g., "Desaprovo-me e faço julgamentos acerca dos meus erros e inadequações"; Humanidade Comum (e.g., "Tento ver os meus erros e falhas como parte da condição humana”); Isolamento (e.g., "Quando penso acerca das minhas inadequações e defeitos sinto-me mais separado e desligado do resto do mundo"); Mindfulness (e.g., "Quando alguma coisa dolorosa acontece tento ter uma visão equilibrada da situação") e Sobreidentificação (e.g., "Quando me sinto em baixo tendo a ficar obcecado com tudo aquilo que está errado").

A versão portuguesa apresenta uma boa consistência interna para o total da escala 0,89 (na versão original americana 0,92), uma boa fidelidade temporal 0,78 (na versão original americana 0,93 ) e boa validade de constructo (Castilho e Pinto-Gouveia, 2011).

A Escala de Satisfação com o Suporte Social (ESSS; Pais Ribeiro, 1999) avalia a satisfação sentida pelo indivíduo relativamente ao suporte social percebido. É constituída por 15 itens que se organizam em 4 fatores: satisfação com os amigos, intimidade, satisfação com a família e atividades sociais, sendo cada item cotado numa escala entre 1 e 5 pontos. O Total da escala revela uma boa consistência interna $(\alpha=0,85)$ e as sub-escalas apresentam valores alfa de Cronbach que variam entre 0,64 (Atividades Sociais) e 0,83 (Satisfação com os Amigos) (Pais Ribeiro, 1999).

A Escala de Depressão, Ansiedade e Stress (Depression, Anxiety and Stress Scale - DASS-21; Lovibond e Lovibond, 1995, EADS-21, versão portuguesa de Pais-Ribeiro, Honrado e Leal, 2004) é composta por 21 itens que avaliam três dimensões de sintomas emocionais negativos: Depressão, Ansiedade e Stress, 
cada uma constituída por 7 itens, utilizando uma escala de resposta de 4 pontos, entre o e 3 .

A versão portuguesa evidenciou valores adequados de consistência interna com valores de alfa de Cronbach que variaram entre 0,74 e 0,85 (Pais-Ribeiro, Honrado e Leal, 2004).

\section{Procedimento}

O projeto de investigação foi submetido à apreciação da Comissão de ética do Centro Hospitalar da Universidade de Coimbra, tendo o mesmo sido aprovado.

Para a recolha de dados, foi aplicada uma bateria de questionários de autorresposta concebida para analisar o papel/impacto da autocompaixão e do suporte social no que respeita ao ajustamento mental ao cancro do pulmão, avaliando ainda os sintomas emocionais negativos: depressão, ansiedade e stress dos participantes.

Os instrumentos foram administrados individualmente, em contexto de gabinete enquanto os doentes se encontravam no serviço a aguardar consultas e/ou a realização de tratamentos, demorando o seu preenchimento cerca de 20 minutos. Previamente ao preenchimento os participantes foram informados acerca dos objetivos do estudo, do facto de a sua participação ser voluntária e de ser assegurada a confidencialidade e anonimato dos dados recolhidos. Todos os participantes deram o seu consentimento informado por escrito.

Os indivíduos que manifestaram grandes dificuldades de ajustamento à doença e/ou níveis significativos de sintomas emocionais negativos, foram, consequentemente, aconselhados/encaminhados para acompanhamento psicológico no serviço, através das consultas de Psico-Oncologia.
Revista Portuguesa de Investigação Comportamental e Social 2016, Vol. 2 (1): 14-24 Portuguese Journal of Behavioral and Social Research 2016, Vol. 2 (1): 14-24

\section{Procedimento Estatístico}

Para o tratamento estatístico, recorreu-se ao software estatístico Statistical Package for the Social Sciences (SPSS), versão 21.0.

A análise da consistência interna dos vários instrumentos medida foi calculada através do método estatístico alfa de Cronbach. Para a comparação de médias entre dois grupos utilizámos o teste $t$ de Student para amostras independentes. O coeficiente de correlação de Pearson foi utilizado para analisar o grau de associação entre variáveis contínuas.

Foi analisado o conjunto de variáveis que melhor prediz o ajustamento mental à doença e aos sintomas emocionais negativos através do modelo de Análise de Regressão Linear. Num primeiro momento, foram averiguados os pressupostos para a realização destas análises, sendo cumprido o critério do tamanho da amostra e da ausência de multicolineridade (todas as variáveis independentes apresentaram valores de tolerância superiores a 0,10 e VIFs inferiores a 10) (Tabachnick e Fidell, 2007).

Não obstante as variáveis não seguirem uma distribuição normal (o pressuposto de normalidade foi analisado através do teste de Kolmogorov-Smirnov), os valores de assimetria e achatamento não evidenciam graves enviesamentos. Segundo Kline (2005), valores de assimetria $|<3|$ e de achatamento $|<10|$ são aceitáveis.

\section{RESULTADOS}

\section{Caracterização clínica da amostra}

As características clínicas da amostra em estudo estão apresentadas na Tabela 1.

Tabela 1

Dados Clínicos da Amostra $(\mathrm{N}=55)$

\begin{tabular}{|c|c|c|c|}
\hline & & \multicolumn{2}{|c|}{ Amostra Total $(N=55)$} \\
\hline & & $N$ & $\%$ \\
\hline \multirow{2}{*}{ Fumador } & Sim & 29 & 52,7 \\
\hline & Não & 26 & 47,3 \\
\hline \multirow{2}{*}{ Antecedente familiar oncológico } & Sim & 25 & 45,5 \\
\hline & Não & 30 & 54,5 \\
\hline \multirow{3}{*}{ Antecedente familiar idêntico } & Sim & 8 & 14,5 \\
\hline & Não & 17 & 30,9 \\
\hline & & $M$ & DP \\
\hline \multicolumn{2}{|l|}{ Tempo de Fumador (meses) } & 394,34 & 170,76 \\
\hline \multicolumn{2}{|l|}{ Conhecimento diagnóstico (meses) } & 25,25 & 41,92 \\
\hline \multicolumn{2}{|l|}{ Perceção da doença } & 2,24 & 0,86 \\
\hline
\end{tabular}


Na avaliação da variável "perceção da doença" referente à ideia que os indivíduos têm acerca da gravidade e evolução da doença, foi utilizada uma escala de resposta de 5 pontos ( 1 = Muito Má; 2 = Má; $3=$ Razoável; $4=$ Boa; 5 = Muito Boa). Constata-se que existem diferenças estatisticamente significativas da perceção da doença em função do sexo [ $t(53)=-2,09, p=$ 0,041 , percecionando as mulheres a situação clínica como mais grave $(M=2,59, D P=0,87)$, comparativamente aos homens $(M=2,08 ; D P=0,82)$.

$\mathrm{Na}$ Tabela 2 encontram-se expostos os valores médios obtidos nos instrumentos de medida. Foram exploradas eventuais diferenças significativas em função do sexo, e apenas a dimensão autocrítica revelou uma diferença estatisticamente significativa $[t(53)=2,10 ; p=$ $0,041]$, com os homens a revelarem valores mais elevados $(M=3,01, D P=0,83)$, comparativamente às mulheres $(M=2,48, D P=0,91)$.

Relativamente à idade e ao tempo de conhecimento acerca do diagnóstico não foram encontradas correlações estatisticamente significativas com nenhuma das variáveis em estudo.

\section{Influência das variáveis clínicas}

Adicionalmente procurámos analisar se algumas variáveis clínicas (e.g., ser ou não fumador, ter ou não antecedentes familiares oncológicos, o tempo de conhecimento de diagnóstico e a perceção da gravidade da doença) poderiam afetar os resultados nos instrumentos de medida (estratégias de coping, suporte social, autocompaixão, e sintomas emocionais negativos).

Relativamente à variável "fumador", verificaram-se diferenças significativas na dimensão Fatalismo (MiniMac) $[t(53)=-2,35 ; p=0,022]$, na subescala autocrítica (SELFCS) [t $(53)=2,13, p=0,038]$, no total da escala da autocompaixão (SELFCS) $[t(53)=-2,28, p=0,027]$, no fator satisfação com os amigos (ESSS) $[t(53)=-2,38 ; p=$ $0,021]$ e na subescala de sintomatologia depressiva (EADS-21) [t $(53)=2,00 ; p=0,050]$. Os fumadores, comparativamente aos não-fumadores, apresentaram menores níveis de fatalismo ( $M=14,31$ vs. $M=15,73)$, são mais autocríticos $(M=3,08$ vs. $M=2,58)$, são menos autocompassivos no geral ( $M=3,28$ vs. $M=3,61)$, sentemse menos satisfeitos com o suporte dos amigos ( $M=18,28$ vs. $M=20,58)$ e apresentam mais sintomatologia depressiva $(M=3,24$ vs. $M=1,65)$.

No que respeita à variável "antecedente familiar oncológico" observaram-se diferenças estatisticamente significativas em relação ao fator intimidade [ESSS: $t$ (53) $=-2,14 ; p=0,037]$, evidenciando os sujeitos com algum tipo de antecedente familiar oncológico níveis mais baixos de "intimidade" no suporte social, comparativamente aos sujeitos sem qualquer antecedente familiar $(M=14,92$ vs. $M=16,70)$.

Tabela 2

Valores Médios e de Consistência Interna (alfa de Cronbach) dos Instrumentos de Medida ( $N=55)$

\begin{tabular}{lccc}
\hline & M & SD & $\boldsymbol{\alpha}$ \\
\hline MiniMac-Desânimo/fraqueza & 14,42 & 4,46 & 0,89 \\
MiniMac-Perturbação Ansiosa & 19,51 & 5,50 & 0,90 \\
MiniMac-Espírito Luta & 12,51 & 1,73 & 0,66 \\
MiniMac-Evitamento Cognitivo & 11,29 & 2,57 & 0,85 \\
MiniMac-Fatalismo & 14,98 & 2,33 & 0,66 \\
Total-Selfcs & 3,49 & 0,67 & 0,92 \\
Selfcs-calor/compreensão & 2,84 & 0,88 & 0,84 \\
Selfcs-Autocrítica & 3,54 & 0,68 & 0,84 \\
Selfcs-Condição Humana & 2,57 & 0,82 & 0,79 \\
Selfcs-Isolamento & 3,65 & 0,64 & 0,73 \\
Selfcs-Mindfulness & 2,78 & 0,89 & 0,82 \\
Selfcs-Sobreidentificação & 19,36 & 3,73 & 0,84 \\
Total-Esss & 15,89 & 3,17 & 0,83 \\
ESSS-SatisfaçãoAmigos & 12,69 & 2,50 & 0,75 \\
ESSS-Intimidade & 9,35 & 2,90 & 0,64 \\
ESSS-SatisfaçãoFamilia & 57,29 & 9,14 & 0,80 \\
ESSS-AtividadesSociais & 2,25 & 2,23 & 0,55 \\
DASS21-Ansiedade & 2,49 & 3,02 & 0,63 \\
DASS21-Depressão & 5,49 & 3,70 & 0,84 \\
DASS21-Stress & 3,44 & 0,56 & 0,79 \\
\hline
\end{tabular}

Notas: MiniMac = Escala de Ajustamento Mental ao Cancro; SELFCS = Self-Compassion Scale; ESSS = Escala de Satisfação com o Suporte Social; DASS = Depression, Anxiety and Stress Scale. 
A variável referente ao tempo que tinham conhecimento do diagnóstico não se mostrou correlacionada com nenhuma das variáveis em estudo ( $p>0,005)$.

Por último, no que toca à variável perceção da gravidade da doença, esta evidencia uma correlação negativa e significativa com as dimensões de Desânimo/ Fraqueza $(r=-0,28 ; p=0,040)$ e Preocupação Ansiosa $(r=$ $-0,45 ; p=0,001)$ da MiniMac. Isto significa que quanto mais grave é percecionada a doença pelo doente, maiores níveis de desânimo/fraqueza e preocupação ansiosa apresenta.

Análise das correlações entre Ajustamento Mental ao Cancro (Desânimo, Preocupação Ansiosa, Evitamento Cognitivo) e Sintomas emocionais negativos (Depressão, Stress) e as restantes variáveis em estudo (dimensões da autocompaixão e do suporte Social)

Na Tabela 3 apresentamos os coeficientes de correlação de Pearson entre as variáveis ajustamento mental ao cancro, autocompaixão, suporte social e sintomas emocionais negativos. Nesta análise, optámos por selecionar apenas as subescalas da MiniMac que evidenciaram consistências internas adequadas (Desânimo/fraqueza, Preocupação Ansiosa e Evitamento Cognitivo: alfa de Cronbach igual a 0,89, 0,90 e 0,85, respetivamente).

Relativamente aos sintomas emocionais negativos, optámos por analisar apenas as dimensões de Depressão e Stress, uma vez que a escala de Ansiedade revelou nesta amostra uma consistência interna abaixo do recomendável.

De acordo com a Tabela 3, podemos observar que a dimensão desânimo/fraqueza encontra-se associada significativamente, de forma positiva e moderada, com a depressão. Por sua vez, mostra uma associação negativa baixa com a subescala de mindfulness, e com o total da escala de suporte social, bem como com as suas dimensões de intimidade e de satisfação com o suporte da família.

Em relação à dimensão preocupação ansiosa, verificaram-se associações positivas e estatisticamente significativas com as medidas de autocrítica, isolamento, sobreidentificação depressão e stress.

Por último, a estratégia de evitamento cognitivo apenas evidenciou uma associação positiva baixa com as dimensões de Calor/compreensão e de Mindfulness (da escala de autocompaixão).

No que respeita aos sintomas emocionais negativos, os sintomas depressivos encontram-se associados de forma positiva e estatisticamente significativa com o isolamento e a sobreidentificação (dimensões da autocompaixão), bem como com o nível de stress, estratégias de desânimo/fraqueza e de preocupação ansiosa. Revelam ainda uma associação negativa com o traço mindfulness, total da escala de suporte social, satisfação com os amigos e satisfação com a família.

Tabela 3

Análise das Correlações entre Outcomes (Dimensões de Desânimo, Preocupação Ansiosa, Evitamento Cognitivo, Depressão e Stress) e as Restantes Variáveis em Estudo (Dimensões da Auto-Compaixão e Suporte Social)

\begin{tabular}{|c|c|c|c|c|c|}
\hline & $\begin{array}{l}\text { MiniMac } \\
\text { Desânimo }\end{array}$ & $\begin{array}{c}\text { MiniMac } \\
\text { Preocupação } \\
\text { Ansiosa }\end{array}$ & $\begin{array}{c}\text { MiniMac } \\
\text { Evitamento } \\
\text { Cognitivo }\end{array}$ & $\begin{array}{c}\text { DASS } \\
\text { Depressão }\end{array}$ & $\begin{array}{l}\text { DASS } \\
\text { Stress }\end{array}$ \\
\hline Total SELFCS & $-0,18$ & $-0,26$ & 0,25 & $-0,28^{*}$ & $-0,26$ \\
\hline Calor/compreensão & $-0,00$ & $-0,02$ & $0,27^{*}$ & $-0,09$ & $-0,00$ \\
\hline Condição Humana & 0,08 & 0,17 & 0,24 & 0,06 & 0,18 \\
\hline Mindfulness & $-0,38 * *$ & $-0,23$ & $0,37^{* *}$ & $-0,41 * *$ & $-0,24$ \\
\hline Autocrítica & 0,02 & $0,29 *$ & $-0,17$ & 0,15 & $0,27^{*}$ \\
\hline Sobreidentificação & 0,19 & $0,39 * *$ & $-0,09$ & $0,31^{*}$ & $0,41 * *$ \\
\hline Isolamento & 0,24 & $0,30 *$ & $-0,04$ & $0,33^{*}$ & $0,31 * *$ \\
\hline Total ESSS & $-0,39 * *$ & $-0,13$ & 0,15 & $-0,29^{*}$ & $-0,51 * * *$ \\
\hline Satisfação Amigos & $-0,20$ & $-0,01$ & 0,17 & $-0,32 * *$ & $-0,48 * * *$ \\
\hline Satisfação Família & $-0,40 * *$ & $-0,07$ & 0,23 & $-0,31^{*}$ & $-0,35 * * *$ \\
\hline Intimidade & $-0,39 * *$ & $-0,06$ & 0,06 & $-0,11$ & $-0,25$ \\
\hline Atividades Sociais & $-0,22$ & $-0,27$ & 0,00 & $-0,13$ & $-0,43 * * *$ \\
\hline DASS - Depressão & $0,57 * * *$ & $0,44 * * *$ & $-0,15$ & - & $0,57^{* * *}$ \\
\hline DASS - Stress & 0,23 & $0,34^{*}$ & $-0,15$ & $0,57 * * *$ & - \\
\hline
\end{tabular}

Notas: SELFCS = Self-Compassion Scale; ESSS = Escala de Satisfação com o Suporte Social; DASS = Depression, Anxiety and Stress Scale; MiniMac = Escala de Ajustamento Mental 
Por último os sintomas de stress mostram-se associados positivamente à sobreidentificação, ao isolamento e à autocrítica, assim como aos sintomas depressivos e estratégia de preocupação ansiosa. Reportam igualmente uma associação negativa com o total da escala de suporte social, e as dimensões de satisfação com amigos, com a família e com as atividades sociais.

\section{Variáveis preditoras do Ajustamento Mental ao Cancro e de sintomas emocionais negativos}

Para compreender qual o conjunto de variáveis que melhor contribui para o ajustamento mental e os sintomas emocionais negativos, foram realizadas análises de regressão linear múltipla, utilizando as dimensões da escala de autocompaixão e o total da satisfação com o suporte social como preditores (variáveis independentes).

Para o ajustamento mental, escolhemos a estratégia de Desânimo/Fraqueza como variável critério (variável independente), uma vez que esta dimensão tem sido uma das mais robustas e consistentes nos diversos estudos da MiniMac (Pais-Ribeiro et al., 2003).

Para a psicopatologia foi selecionada a depressão e o stress como variável dependente. Nos vários modelos de regressão, tendo em conta o tamanho da amostra, apenas foram consideradas as variáveis independentes que, numa análise exploratória se tinham mostrado associadas à variável dependente (ver Tabela 3).

Assim, no caso da estratégia de Desânimo/Fraqueza, (Tabela 4), o modelo de predição foi significativo $\left[R^{2}=\right.$ $0,26 ; F(2,52)=9,04 ; p<0,001]$, explicando as variáveis preditoras $26 \%$ da variância. A satisfação com o suporte social na globalidade e as competências de mindfulness foram ambos preditores significativos.

Tabela 4

Análise de Regressão Múltipla Utilizando as Dimensões da Autocompaixão (SELFCS) e o Total da Satisfação com o Suporte Social (ESSS) para Predizer a Estratégia de Desânimo/fraqueza (MINIMAC) em Doentes com Cancro do Pulmão $(N=55)$

\begin{tabular}{lccc}
\hline & \multicolumn{3}{c}{ Desânimo/fraqueza (MiniMac) } \\
\hline & $\boldsymbol{R}^{\mathbf{2}}$ & $\boldsymbol{\beta}$ & $\boldsymbol{P}$ \\
\hline SELFCS - Mindfulness & 0,26 & & \\
ESSS - Suporte social global & & $-0,33$ & $\mathbf{0 , 0 1 0}$ \\
\end{tabular}

Notas: SELFCS = Self-Compassion Scale; ESSS = Escala de Satisfação com o Suporte Social; MiniMac = Escala Reduzida de Ajustamento Mental ao Cancro.

Na predição da depressão (Tabela 5), o modelo foi significativo $\left[R^{2}=0,23 ; F(4,50)=3,71 ; p=0,010\right]$, explicando as variáveis preditoras $23 \%$ da variância.

As competências de mindfulness emergiram como o único preditor global.
Tabela 5

Análise de Regressão Múltipla Utilizando as Dimensões da Autocompaixão (SELFCS) e o Total da Satisfação com o Suporte Social (ESSS) para Predizer a Sintomatologia Depressiva (DASS-21) em Doentes com Cancro do Pulmão $(N=55)$

\begin{tabular}{lccc}
\hline & \multicolumn{3}{c}{ Depressão (DASS-21) } \\
\hline & $\boldsymbol{R}^{2}$ & $\boldsymbol{\beta}$ & $\boldsymbol{p}$ \\
\hline & 0,23 & & \\
SELFCS - Mindfulness & & $-0,34$ & $\mathbf{0 , 0 3 2}$ \\
SELFCS - Isolamento & & 0,15 & 0,422 \\
SELFCS - Sobreidentificação & & $-0,06$ & 0,768 \\
ESSS - Suporte social global & & $-0,20$ & 0,144 \\
\hline
\end{tabular}

Notas: SELFCS = Self-Compassion Scale; ESSS = Escala de Satisfação com o Suporte Social; DASS = Depression, Anxiety and Stress Scale.

No modelo preditivo para o stress (Tabela 6), os resultados evidenciaram um modelo significativo $\left[R^{2}=\right.$ 0,$35 ; F(4,50)=6,78 ; p<0,001$ ] que explica $35 \%$, mostrando-se a variável satisfação com o suporte social o melhor preditor $(\beta=-0,46)$ seguido da dimensão de sobreidentificação $(\beta=0,42)$.

Tabela 6

Análise de Regressão Múltipla Utilizando as Dimensões da Autocompaixão (SELFCS) e o Total da Satisfação com o Suporte Social (ESSS) para Predizer a Sintomatologia Associada ao Stress (DASS-21) em Doentes com Cancro do Pulmão $(N=55)$

\begin{tabular}{lccc}
\hline & \multicolumn{3}{c}{ Stress (DASS-21) } \\
\hline & $\boldsymbol{R}^{\mathbf{2}}$ & $\boldsymbol{\beta}$ & $\boldsymbol{p}$ \\
\hline & 0,35 & & \\
SELFCS - Autocrítica & & $-0,04$ & 0,806 \\
SELFCS - Isolamento & $-0,14$ & 0,444 \\
SELFCS - Sobreidentificação & & 0,42 & 0,042 \\
ESSS - Suporte social global & & $-0,46$ & $\mathbf{0 , 0 0 1}$ \\
\hline
\end{tabular}

Notas: SELFCS = Self-Compassion Scale; ESSS = Escala de Satisfação com o Suporte Social; DASS = Depression, Anxiety and Stress Scale.

\section{DISCUSSÃO}

O presente estudo teve como objetivo primordial analisar o papel/impacto da autocompaixão e do suporte social no ajustamento mental ao cancro do pulmão e nos sintomas emocionais negativos associados. Procurou-se igualmente explorar o papel do género e de variáveis clínicas sobre as dimensões anteriormente referidas.

Começando pela análise das características demográficas e clínicas da nossa amostra, verifica-se que a distribuição da doença por sexo foi semelhante à encontrada nos estudos de Alves, Bastos e Lunet (2009) que descreveram valores de $80 \%$ de incidência da doença no sexo masculino e $20 \%$ no sexo feminino. 
Relativamente ao meio de residência dos participantes, o mais frequente é o meio rural, ainda que pouco expectável, uma vez que o meio urbano está associado a maiores níveis de poluição e stress, aspetos esses frequentemente aliados ao desenvolvimento do cancro do pulmão. Contudo, estes resultados podem estar relacionados com outros fatores de risco para esta doença, tais como a predisposição genética ou a radiação e outros agentes químicos a que os indivíduos, ao longo de muito tempo, pudessem estar expostos (Alberg e Samet, 2003). Ainda que não se tenha procedido a uma recolha sistematizada de informação acerca da profissão, durante a administração dos instrumentos de avaliação, foi possível constatar através dos relatos dos participantes que a profissão de alguns esteve associada à exposição de gases, pós e outros agentes químicos e radioativos, o que está em consonância com a revisão da literatura.

Quanto à perceção da gravidade da doença, verificou-se que o sexo masculino tem melhor perceção acerca do diagnóstico e evolução da doença, sendo que as mulheres têm maiores receios e uma visão geral mais negativista.

O presente estudo revelou um número baixo de fumadores diagnosticados com neoplasia pulmonar, comparativamente com outros estudos, tais como os de Alberg e Samet (2003) que afirmaram que 90\% dos casos de cancro do pulmão estavam ligados ao consumo de tabaco. Por outro lado, os resultados revelados no nosso estudo referem-se a fumadores ativos, o que significa que, de entre os indivíduos não fumadores, muitos podem ter sido fumadores. Foram vários os participantes que consideraram terem sido por longos anos fumadores passivos entre as suas rotinas diárias e o seu próprio local de trabalho, dando a maioria os escritórios como exemplo.

Em relação à eventual influência das variáveis clínicas (e.g., ser ou não fumador, ter ou não antecedentes familiares oncológicos, o tempo de conhecimento de diagnóstico, e a perceção da gravidade da doença) no ajustamento mental ao cancro, autocompaixão, suporte social percebido e sintomas emocionais negativos, verificaram-se algumas associações relevantes. Os indivíduos fumadores, comparativamente aos não-fumadores, apresentam menores níveis de fatalismo (atitudes ausentes, serenidade sobre o diagnóstico e aceitação passiva), são mais autocríticos (o que pode estar associado ao sentimento de culpabilidade pela exposição a fatores de elevado risco), são menos autocompassivos no geral (menos calorosos, tolerantes e aceitantes das suas experiências internas e externas), sentem-se menos satisfeitos com o suporte dos amigos (o que pode estar relacionado com o facto de, normalmente, os amigos também serem fumadores e portanto acharem mais fácil terem adotado esse comportamento) e apresentam mais sintomatologia depressiva (frequentemente associada à elevada culpabilidade sentida).

No que respeita à variável "antecedente familiar oncológico", verificou-se que os sujeitos com algum tipo de antecedente familiar oncológico apresentavam níveis mais baixos de "intimidade" nas relações sociais, ou seja, menor satisfação com o suporte social íntimo.

A variável perceção da gravidade da doença evidenciou, como esperado, uma correlação significativa com as estratégias de coping de Desânimo/Fraqueza e Preocupação Ansiosa, mostrando que quanto mais grave é percecionada a doença pelo doente, maiores níveis de desânimo/fraqueza e preocupação ansiosa apresenta.

No que toca à forma como as variáveis em estudo (ajustamento mental, suporte social autocompaixão e sintomas emocionais negativos) estão associadas entre si, os resultados mostraram-se em consonância com as nossas expectativas iniciais. A dimensão desânimo/ fraqueza encontra-se associada de forma positiva com a depressão, o que significa que quantos maiores são os níveis de pessimismo e sentido de perda de saúde ou mesmo perda de controlo da doença, maiores são também os níveis de sintomatologia depressiva. Por outro lado, esta dimensão está ainda associada de forma negativa com as competências de mindfulness, e com a satisfação global com o suporte social, nomeadamente com a satisfação com o grau de intimidade das relações e satisfação com a família. Isto é, quanto maior for o sentimento de pessimismo e perda de controlo da doença, menor é a aceitação sem julgamento das nossas experiências, menor é a perceção de ter alguém verdadeiramente íntimo com quem contar e menor é a satisfação sentida em relação à família.

Quanto à subescala perturbação ansiosa, verificaram-se associações positivas e significativas em relação às subescalas autocrítica, isolamento, sobreidentificação, ansiedade, depressão e stress. Assim sendo, indivíduos marcados por uma ansiedade persistente que procuram informação compulsivamente como resposta comportamental sobre o cancro, são indivíduos que se revelam mais autocríticos, com tendência a procurar o isolamento, a sobreidentificaremse com os seus pensamentos e sentimentos e apresentam maior sintomatologia ansiosa, depressiva e associada ao stress.

Relativamente aos sintomas emocionais negativos referidos pela nossa amostra (ansiedade, depressão e stress), os resultados evidenciaram correlações significativas e no sentido esperado com as medidas em estudo. Mais concretamente a depressão e o stress mostraram-se associados positivamente a estratégias de coping negativo ou de desajustamento (estratégia de Desânimo/fraqueza e Preocupação Ansiosa) e a mecanismos emocionais ineficazes (isolamento e sobreidentificação com o sofrimento). Inversamente, 
evidenciaram associações negativas com as estratégias consideradas positivas ou facilitadoras de um bom ajustamento (compaixão global, mindfulness, suporte social global, satisfação com o suporte dos amigos, e da família). Estes resultados são, em parte, consonantes com outros estudos que apontam esta relação entre sintomas emocionais (positivos/negativos) e estratégias de regulação emocional positivas (mindfulness, calor/ compreensão, condição humana) e negativas (sobreidentificação, isolamento, autocrítica) (PintoGouveia, Duarte, Matos e Fráguas, 2013; Leary, Tate, Adams, Allen e Hancock, 2007; Neff e Costigan, 2014). Outros estudos encontraram também o mesmo tipo de padrão de associação entre as estratégias negativas de ajustamento à doença (MiniMac) e a satisfação com o suporte social (Santos, Ribeiro e Lopes, 2003).

Finalmente, quando é explorado qual o melhor modelo preditor do ajustamento mental à doença e da sintomatologia depressiva e associada ao stress em doentes com cancro, os dados revelaram o contributo diferencial do mindfulness, satisfação global com o suporte social e da sobreidentificação, consoante os modelos em análise. Enquanto para a estratégia de desânimo, a satisfação global com o suporte social e as competências de mindfulness parecem ter um contributo significativo, para a sintomatologia depressiva, o mindfulness aparece como único preditor. Assim, parece que desenvolver um estado de autoconsciência, de atenção plena focada no presente e de aceitação sem julgamento das nossas experiências poderá atenuar a intensidade de estados emocionais negativos, o que está de acordo com a revisão da literatura sobre os benefícios da prática de mindfulness (Baer, 2003; Brown e Ryan, 2003). Por sua vez, o grau de satisfação com o suporte social na globalidade e a não sobreidentificação com o sofrimento revelaram ser contributos significativos na predição do stress, mostrando que quanto maior é a satisfação com apoio dado pelos outros (amigos, família, comunidade) e maior a capacidade de se descentrar do sofrimento, não se fusionando e aceitando-o sem o julgar, menor é o stress sentido por estes doentes oncológicos.

\section{Limites e pesquisas futuras}

Uma das limitações prende-se com a representatividade da amostra dada a sua dimensão reduzida, sendo útil replicar este estudo numa amostra maior. De referir ainda que foram incluídos doentes em diferentes estádios da sua doença oncológica de modo a assegurar um maior número de participantes num intervalo de tempo diminuto. Assim, futuros estudos deverão ser desenhados de forma a controlar esta variável.

Outra limitação prende-se com a natureza transversal do estudo, não permitindo estabelecer relações causais entre as variáveis, sendo imprescindível outro tipo de estudos que recorram a desenhos longitudinais ou experimentais.

Os instrumentos de avaliação utilizados também podem ter levantado dificuldades, já que alguns dos indicadores da Escala de Ajustamento Mental ficaram aquém do que é desejável em termos da sua fidedignidade. Por outro lado, seria importante utilizar outro tipo de instrumentos que permitissem abordar outros constructos teóricos como a culpabilidade, bem como entrevistas que possibilitassem clarificar alguns dos constructos avaliados.

\section{CONCLUSÃO}

De acordo com a literatura, são escassos os estudos que visam analisar a adaptação mental dos doentes ao cancro do pulmão. No entanto, uma vez que a nível europeu a neoplasia pulmonar é a terceira causa de morte por doença oncológica e sendo, em Portugal, a principal causa de morte por cancro, é crucial uma maior investigação desta condição, nomeadamente a nível psicológico de forma a oferecer um apoio mais eficaz e melhores condições a estes indivíduos para lidar com a doença.

O presente estudo procurou acrescentar à literatura existente informação sobre o papel da autocompaixão (enquanto processo de regulação emocional) e do suporte social no ajustamento mental e sintomas emocionais negativos destes doentes. Ao nível da prática clínica, os nossos resultados sugerem que a intervenção psicológica dirigida a estes pacientes poderá beneficiar com a integração do desenvolvimento de competências autocompassivas e de programas que promovam a qualidade das suas redes sociais, já que estas variáveis se mostraram associadas ao ajustamento mental destes indivíduos.

Conflito de interesses | Conflict of interest: nenhum | none. Fontes de financiamento | Funding sources: nenhuma | none.

\section{REFERÊNCIAS}

Alberg, A. J. e Samet, J. M. (2003). Epidemiology of lung cancer. Chest, 123(Supl. 1), 21S-49S. Acedido em http://www.ncbi.nlm.nih.gov/ pubmed/23649439

Alves L, Bastos J. e Lunet N. (2009). Evolução da mortalidade por cancro do pulmão em Portugal (1955-2005). Revista Portuguesa Pneumologia, 15, 575-87.

Baer, R. A. (2003). Mindfulness training as a clinical intervention: A conceptual and empirical review. Clinical Psychology: Science and Practice, 10, 125-143.

Batista, R., Cunha, M., Galhardo, A., Couto, M. e Massano-Cardoso, I. (2015). European Psychiatry, 30 (Supl. 1), 1511-1511. 1p. Doi: 10.1016/ Sog24-9338(15)31168-8 
Bower, M. e Waxman, J. (2006). Compêndio de oncologia. Lisboa: Instituto Piaget: Coleção Medicina e saúde.

Brown, K. W. e Ryan, R. M. (2003). The benefits of being present: Mindfulness and its role in psychological well-being. Journal of Personality and Social Psychology, 84(4), 822-848.

Castilho, P. e Pinto-Gouveia, J. (2011). Autocompaixão: Estudo da validação da versão portuguesa da Escala da Autocompaixão e da sua relação com as experiências adversas na infância, a comparação social e a psicopatologia. Psychologica, 54, 203-230.

Custódio, S. (2010). Stress, suporte social, otimismo e saúde em estudantes de enfermagem em ensino clínico. Acedido em http://ria.ua.pt/handle/ 10773/1122

Domingues, V. e Albuquerque, E. (2008). Cancro do pulmão: Aspetos psicológicos e psiquiátricos. Revista Portuguesa de Pneumologia, XIV, 261-269.

Ferlay J., Shin H. R., Bray F., Forman D., Mathers C. e Parkin D. M. (2008). Cancer Incidence and Mortality Worldwide: IARC Cancer Base No. 10 (2). Lyon, France: International Agency for Research on Cancer; 2010. Acedido em http://globocan.iarc.fr

Kline, R. B. (2005). Principles and Practice of Structural Equation Modeling ( $2^{\mathrm{a}}$ ed.). New York: Guilford.

Leary, M. R., Tate, E. B., Adams, C. E., Allen, A. B. e Hancock, J. (2007). Self-compassion and reactions to unpleasant self-relevant events: the implications of treating oneself kindly. Journal of personality and social psychology, 92(5), 887-904. Doi: 10.1037/0022-3514.92.5.887

Lovibond, P. e Lovibond, S. (1995). The structure of negative emotional states: Comparison of the depression anxiety stress scales (EADS) with the Beck Depression and Anxiety Inventories. Behaviour Research and Therapy, 33(3), 335-343.

Marques, A. R., Santos, G., Firmino, H., Santos, Z., Vale, L. ... Morais, I. (1991). Reações emocionais à doença grave: Como lidar... Coimbra. Edição Psiquiatria Clínica.

Neff, K. D. (2003a). The development and validation of a scale to measure self-compassion. Self and Identity, 2, 223-250. Doi: 10.1080/15298860390209035

Neff, K. (2003b). Self-compassion: An alternative conceptualization of a healthy attitude toward oneself. Self and Identity, 2, 85-101. Doi: 10.1080/15298860390129863

Neff, K. D. e Costigan, A. P. (2014). Self-compassion, well-being, and happiness. Psychologie in Österreich, 114-117.

Neff, K. D., Kirkpatrick, K. e Rude, S. S. (2007). Self-compassion and its link to adaptive psychological functioning. Journal of Research in Personality, 41, 139-154.

Neff, K. D., Rude, S. S. e Kirkpatrick, K. (2007). An examination of selfcompassion in relation to positive psychological functioning and personality traits. Journal of Research in Personality, 41, 908-916.

Ogden, J. (1999). Psicologia da saúde. Lisboa: Climepsi Editores.

Pais-Ribeiro, J. L. (1999). Escala de satisfação com o suporte social. Análise Psicológica, 3(12), 547-558.

Pais-Ribeiro, J., Honrado, A. e Leal, I. (2004). Contribuição para o estudo da adaptação portuguesa das escalas de Depressão Ansiedade Stress de Lovibond e Lovibond. Psychologica, 36, 235-246.
Revista Portuguesa de Investigação Comportamental e Social 2016, Vol. 2 (1): 14-24 Portuguese Journal of Behavioral and Social Research 2016, Vol. 2 (1): 14-24

Pais-Ribeiro, J. L., Ramos, D. e Samico, S. (2003). Contribuição para uma validação conservadora da escala reduzida de ajustamento mental ao cancro (Mini-MAC). Psicologia, Saúde e Doenças, 4(2), 231-247.

Parente, B., Barata, F. J., Neto, I. G. e Costa, A. (2002). Cancro do pulmão - O doente terminal. Revista Portuguesa de Pneumologia, VIII(4), $351-372$.

Parente, B., Queiroga, H., Teixeira, E., Sotto-Mayor, R., Barata, F., Sousa, A. ... Araújo, A. (2007). Estudo epidemiológico do cancro do pulmão em Portugal nos anos de 2000/2002. Revista Portuguesa de Pneumologia, 13(2), 255-265. Acedido em http:// w w w.scielo.oces.mctes.pt/scielo.ph p ? pid $=$ S087321592007000200005\&script=sci_arttext

Pereira, M. e Lopes, C. (2005). O doente oncológico e sua família. ( $2^{\mathrm{a}}$ ed.) Manuais Universitários: Climepsi.

Pinto-Gouveia, J., Duarte, C., Matos, M. e Fráguas, S. (2013). The Protective Role of Self-compassion in Relation to Psychopathology Symptoms and Quality of Life in Chronic and in Cancer Patients. Clinical Psychology \& Psychotherapy, 21(4), 311-323. doi:10.1002/cpp. 1838

Ribeiro, J. (1999). Escala de satisfação com o suporte social (ESSS). Análise Psicológica, 3(17), 547-558. Acedido em http:// w w w. scielo.oce s.mctes.pt/s cielo.ph p ? pid=S087082311999000300010\&script=sci_arttext

Rodrigues, J. e Ferreira, N. (2012). Estrutura e funcionalidade da rede de apoio social do adulto com câncer. Acta Paulista de Enfermagem, 25(5), 781-787. Retrieved from http://www.scielo.br/pdf/ape/ v25n5/21.pdf

Santos, C. e Pais-Ribeiro, J. (2001). Qualidade de vida do doente oncológico- Revisão da bibliografia. Referência, 7, 5-16;

Santos, C. S. V. de B., Ribeiro, J. P. e Lopes, C. (2003). Estudo de adaptação da Escala de Satisfação com o Suporte Social (ESSS). Psicologia, Saúde \& Doenças, 4(2), 185-204.

Stewart, M., Craig, D., MacPherson, K. e Alexander, S (2001). Promoting positive affect and diminishing loneliness of widowed seniors through a support intervention. Public Health Nursing, 18(1), 54-63.

Stommel, M., Kurtz, M. E., Kurtz, J., Given, C. W. e Given, B. A.. (2004). A longitudinal analysis of the course of depressive symptomatology in geriatric patients with cancer of the breast, colon, lung or prostate. Health Psychology, 23(6), 564-573.

Straub, R. (2005). Psicologia da saúde. Porto Alegre: Artes Médicas.

Tabachnick, B. e Fidell, L. (2007). Using multivariate statistics. New York: Pearson Education Inc.

Usta, Y. Y. (2012). Importance of Social Support in Cancer Patients. Asian Pacific Journal of Cancer Prevention, 13(1976), 3569-3572.

Watson, M., Law, M., Santos, M., Greer, S., Baruch, J. e Bliss, J. (1994). The mini-MAC: Further development of the mental adjustment to cancer scale. Journal of Psychosocial Oncology, 12(3), 33-46.

Zamboni, M. (2002). Epidemiologia do câncer do pulmão. Jornal Pneumologia, 28(1), 41-47. Acedido em http://www.scielo.br/pdf/\%oD/ jpneu/v28n1/ao8v28n1.pdf 